\title{
A comparison of CD105 and CD31 expression in tumor vessels of hepatocellular carcinoma by tissue microarray and flow cytometry
}

\author{
HONGYAN QIAN, LIPING YANG, WENJING ZHAO, HAIZHEN CHEN and SONG HE
}

\author{
Key Laboratory of Cancer Research Center Nantong, Tumor Hospital Affiliated to Nantong University, \\ Nantong, Jiangsu 226361, P.R. China
}

Received December 28, 2017; Accepted July 19, 2018

DOI: $10.3892 /$ etm.2018.6553

\begin{abstract}
Tumor endothelial cells (TECs) have been isolated from solid tumors by using immunological magnetic beads and magnetic active cell sorting, and lead to a more precise way to investigate tumor angiogenesis as well as screening of vascular targeting drugs. However, the question of which endothelial marker is a stable molecular signature in TECs and can be used for the isolation of TECs from tumor tissues remains unclear. In this study, we investigated the endothelial markers CD105 and CD31 in the tumor vessels from 90 patients with hepatocellular carcinoma (HCC) by tissue microarray, in addition to their expression in TECs isolated from fresh tissues resected from 11 patients with HCC by flow cytometry and confocal microscopy. The results revealed that among 90 cases of TMA, all tumor vessels were CD31 positive whereas 39 cases (43.3\%) had little or no CD105 expression in tumors and their vessels but not peritumoral tissue spots, and that among these 39, 29 cases (74.4\%) were poor-differentiated HCC. These findings were further verified by flow cytometry and confocal analysis of TECs isolated from HCC. Overall, the results suggested that CD105 may not be expressed in TECs derived from poor-differentiated HCC cases. In addition, combined with previous studies in which CD105 is not only expressed in TECs, but also in tumor cells, the results indicated a high risk of contamination with $\mathrm{CD} 105^{+}$tumor cells. Thus, there is a limitation to the use CD105 as an endothelial marker for the isolation of TECs.
\end{abstract}

\section{Introduction}

It is well known that tumor angiogenesis, which is the formation of new blood vessels in tumors, has a critical role in tumor progression and metastasis (1). The heterogeneity of tumor vessels has increasingly been demonstrated. It has been reported

Correspondence to: Professor Liping Yang, Key Laboratory of Cancer Research Center Nantong, Tumor Hospital Affiliated to Nantong University, 30 Tongyang North Road, Pingchao, Nantong, Jiangsu 226361, P.R. China

E-mail: liping.yang@ntu.edu.cn

Key words: CD105, CD31, tumor endothelial cells, tissue microarray, flow cytometer, hepatocellular carcinoma that tumor microvascular architecture phenotype (T-MAP), in addition to various bio-characteristics, exhibit heterogeneity and differ from the normal blood vessels $(2,3)$. Thus, using normal vascular endothelial cells (ECs) is inappropriate to investigate tumor angiogenesis or screen candidate anti-cancer drugs that target tumor vessels. It is necessary to harvest tumor endothelial cells (TECs) from tumor tissues. Recently, with the method of magnetic active cell sorting (MACS) that is based on the immunological magnetic beads conjugating various antibodies specifically to endothelial markers such as CD105, CD31 and CD34, many laboratories have made it possible to isolate and purify TECs from tumor mass (4-7). Of these markers, studies have shown that CD105 is a good marker for tumor angiogenesis in endometrial carcinoma, cervical cancer, breast carcinoma, glioblastoma and esophageal squamous cell carcinoma (8-11). However, it has been found that CD105 expression levels in tumor vessels vary with cancer development (12-14).

CD105 is a transmembrane glycoprotein expressed primarily in ECs and tumor cells $(15,16)$. Tumoral CD105 has been defined as a novel independent prognostic marker, whereas the microvessel density labelled by the endothelial marker CD105 (MVD-CD105) negatively correlates with tumor development of human hepatocellular carcinoma (HCC) and renal cell carcinoma $(13,17)$. However, there are conflicting studies as to whether MVD-CD105 is a biological marker for predicting prognosis of cancer. A contrary example is that a higher score of MVD-CD105 appears to correlate with a significantly poorer prognosis in survival rate (14). Furthermore, CD105 exhibits a regulatory role in normal human vascular endothelial cells (HUVECs) $(18,19)$.

The aforementioned experimental findings suggest that CD105 expression levels in TECs exist in high and low states, depending on tumor stages. CD31 (PECAM-1), a well-known pan marker for endothelial cells, has also been used for isolation of TECs (20). In our laboratory we verified that CD31 is a reliable endothelial marker by phenotypic and functional assays (unpublished data). Thus, in the present study, we applied several approaches to detect CD105 and CD31 expression throughout human HCC tissues with various differentiation status and explored the association between CD105 negative expression in TECs and HCC status using a wide range of samples. CD $31^{+}$TECs derived from HCC (termed ECDHCC) were isolated and CD105 expression was analyzed in these cells using flow cytometry and confocal microscopy. 


\section{Materials and methods}

Patients and tissue microarray. All procedures of this study involving human materials were performed according to the ethical standards with the Helsinki Declaration and the China Ministry of Health's 'Ethical Review of Human Biomedical Research (Tentative, 2007)'. The study was approved by the Research Ethics Board of the Tumor Hospital Affiliated to Nantong University. The written informed consent was obtained as specified in the ethical approval.

We retrospectively collected formalin-fixed paraffinembedded (FFPE) tissues from $90 \mathrm{HCC}$ patients with the complete clinicopathological data from January 2003 to December 2006. The diagnosis had been done by two pathologists who were blinded to the clinicopathological data at the Tumor Hospital Affiliated to Nantong University. Clinical follow-up data were retrieved from patient records at the Department of Epidemiology in the Tumor Hospital. All underwent hepatic surgical resection without postoperative systemic chemotherapy in the Surgery Department. The main clinical characteristics of the patients are shown in Table I. 78 patients were men and 12 were women. Histological grades were classified to well differentiated $(\mathrm{n}=38)$, and poorly differentiated ( $n=52)$. Seventy-three were positive for cirrhosis. We prepared 90 pairs (tumor vs. peritumoral tissues) of tissue microarray (TMA) from the aforementioned FFPE tissues. Each patient's specimen was represented by a single $1 \mathrm{~mm}$ core of tissue. The 90 paired TMAs were used for immunohistochemistry (IHC) staining to detect the expression of CD105 and CD31.

In order to detect endothelial maker expression levels in cells derived from HCC by flow cytometry and confocal microscopic analysis, we collected additional fresh resections, which contained less necrosis tissues, from 15 HCC patients at Tumor Hospital Affiliated to Nantong University from January to December of 2016 with the written informed consent and ethical approval by Research Ethics Committee of Tumor Hospital Affiliated to Nantong University. The clinical characteristics of the patients are shown in Table I. These fresh samples were from 6 male and 9 female patients with 35-75 year of age. Histological grades were classified to well differentiated $(n=8)$, and poorly differentiated $(n=7)$. The diagnosis had been done by two pathologists who were blinded to the clinicopathological data and clinical follow-up data were retrieved from patient records at the Department of Epidemiology in the aforementioned hospital. Single cell suspension was prepared for fluorescent antibody staining. We used these 15 paired samples to check the markers, particularly CD105 expression in tumors vs. peritumoral areas. A positive expression vs. a negative expression in the matched pairs was verified as a convincing result.

IHC staining and analysis. FFPE slices were dewaxed in xylene and rehydrated in graded alcohol. For blocking of endogenous peroxides, $3 \%$ hydrogen peroxide was used for $15 \mathrm{~min}$. Antigen retrieval was routinely performed by immersing the slices in a thermostatic bath containing preheated ethylene diamine tetra acetic acid (EDTA) for $30 \mathrm{~min}$ at $98^{\circ} \mathrm{C}$ and cooling down at room temperature for $20 \mathrm{~min}$. Sections were incubated with monoclonal antibody against a 1:30 dilution of
Table I. Clinical characteristics of 90 paired FFPE and 15 fresh tissues from patients with HCC.

\begin{tabular}{|c|c|c|}
\hline Parameter & $\begin{array}{c}\text { FFPE tissues } \\
\mathrm{n}=90(\%)\end{array}$ & $\begin{array}{c}\text { Fresh tissues } \\
\mathrm{n}=15(\%)\end{array}$ \\
\hline \multicolumn{3}{|l|}{ Age, years } \\
\hline$\leq 45$ & $33(36.7)$ & $5(33.3)$ \\
\hline$>45$ & $57(63.3)$ & $10(66.7)$ \\
\hline \multicolumn{3}{|l|}{ Sex } \\
\hline Male & 78 (86.7) & $6(40.0)$ \\
\hline Female & $12(13.3)$ & $9(60.0)$ \\
\hline \multicolumn{3}{|c|}{ Tumor differentiation } \\
\hline Well & $38(42.2)$ & $8(53.3)$ \\
\hline Poor & $52(57.8)$ & $7(46.7)$ \\
\hline \multicolumn{3}{|c|}{ Tumor size, cm } \\
\hline$\leq 5$ & $51(56.7)$ & $8(53.3)$ \\
\hline$>5$ & $39(43.3)$ & $7(46.7)$ \\
\hline \multicolumn{3}{|c|}{ Capsular integrity } \\
\hline Positive & $57(63.3)$ & $9(60.0)$ \\
\hline Negative & $33(36.7)$ & $6(40.0)$ \\
\hline \multicolumn{3}{|l|}{ Metastasis } \\
\hline Positive & $14(15.6)$ & $3(20.0)$ \\
\hline Negative & $76(84.4)$ & $12(80.0)$ \\
\hline \multicolumn{3}{|c|}{ Vascular invasion } \\
\hline Positive & $67(74.4)$ & $7(46.7)$ \\
\hline Negative & $23(25.6)$ & $8(53.3)$ \\
\hline \multicolumn{3}{|c|}{ Liver cirrhosis } \\
\hline Positive & $73(81.1)$ & $11(73.3)$ \\
\hline Negative & $17(18.9)$ & $4(26.7)$ \\
\hline \multicolumn{3}{|l|}{ AFP, ng/ml } \\
\hline$\leq 50$ & $34(37.8)$ & $6(40.0)$ \\
\hline$>50$ & $56(62.2)$ & $9(60.0)$ \\
\hline
\end{tabular}

FFPE, formalin-fixed paraffin-embedded; HCC, hepatocellular carcinoma; AFP, $\alpha$ fetoprotein. $n$, the number of the case.

CD105 (mouse-anti-human, clone SN6 h; DAKO, Glostrup, Denmark) and a 1:50 dilution of CD31 (mouse-anti-human, clone JC70A) (both from DAKO, Glostrup, Denmark) overnight at $4^{\circ} \mathrm{C}$. Visualization of the antibody complex was achieved with a diaminobenzidine (DAKO) reaction, resulting in brown staining of EC membranes. TMA and slices were counterstained by Meyer's hematoxylin.

We used both qualitative and quantitative analysis for evaluation of IHC results. By qualitative analysis, 'negative expression' was indicated by the average number of positive cells from 3 hot spots covering $<5 \%$ of the total cells. For quantitative analysis, we used average optical density (AOD) to evaluate the intensity of the IHC reaction in order to compare expression levels between tumor and peritumoral fields of different markers. AOD was performed by Image-Proplus 6.0 software. AOD scores were calculated from the optical density of 5 spots selected randomly on each slice under the microscope $(x 400)$. 


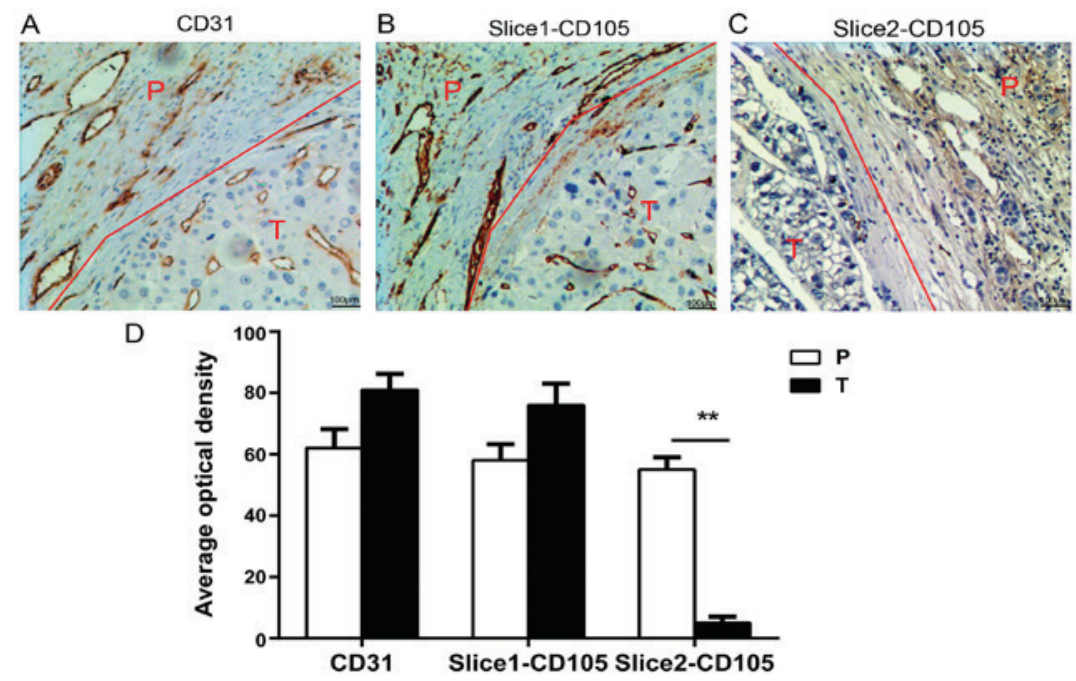

Figure 1. Endothelial marker expression in vessels of HCC tissues. Immunohistochemistry staining of CD31 and CD105 was carried out in 15 fresh HCC tissues. (A) Representative image of CD31 expression in tumor and peritumoral tissues. Representative images of (B) CD105 positive (slice1-CD105) or (C) CD105 negative (slice2-CD105) expression in tumor tissues, respectively. (D) Average optical density (AOD) of CD31 (n=15), CD105 (positive, n=9) and CD105 (negative, $\mathrm{n}=6$ ). Magnification $\mathrm{x} 100$. T, tumor, $\mathrm{P}$, peritumoral tissues. ${ }^{* *} \mathrm{P}<0.01$.

Isolation of TECs from HCC. A single cell suspension was firstly prepared from the fresh surgical specimens of the patients with HCC. Briefly, the specimens were minced with scissors and digested by incubation in HANK'S medium (containing $\mathrm{Ca}^{2+}, \mathrm{Mg}^{2+}$ ) supplemented with $0.1 \%$ collagenase I and collagenase IV (Sigma-Aldrich; Merck KGaA, Darmstadt, Germany), and DNase (Cell Culture Grade; Roche Diagnostics, Basel, Switzerland) at $37^{\circ} \mathrm{C}$ for $45 \mathrm{~min}$. After being washed in medium plus $10 \%$ FBS (Sijiqing Co., Ltd., Hangzhou, China), the cells were ready for MACS separation. We used anti-CD31 antibody coupled to magnetic beads and MACS system (Miltenyi Biotech, Bergisch Gladbach, Germany) to separate CD $31^{+}$TECs from other cells in the cell suspension, according to the instruction manual.

Cell lines and culture conditions. Normal vascular endothelial cell line (HUVEC) and isolated ECDHCC cells were maintained in endothelial cell growth medium (ECM) supplemented with $5 \%$ fetal bovine serum (FBS), 2\% VEGF, $100 \mathrm{U} / \mathrm{ml}$ penicillin and $100 \mathrm{U} / \mathrm{ml}$ streptomycin in $5 \% \mathrm{CO}_{2}$. All these, including HUVEC cell line were purchased from ScienCell Research Laboratory (Yuhenfeng Company, Beijing, China).

Flow cytometric analysis. The first step was the preparation of a single cell suspension. The cultured HUVEC cells were detached from plates with a nonenzymatic cell dissociation solution (Sigma-Aldrich; Merck KGaA) and washed in PBS containing $0.5 \%$ BSA. The fresh surgical specimens were enzymatically dissociated into a single cell suspension. Cells were then incubated for $15 \mathrm{~min}$ at $4^{\circ} \mathrm{C}$ with the appropriate antibodies or with a control in PBS containing $0.5 \%$ bovine serum albumin (BSA). Cells were analyzed on Becton Dickinson FACS Aril II. We used primary murine monoclonal antibodies against human CD105 conjugated to allophycocyanin (APC) (1:30 dilutions) and human CD31 conjugated to phycoerythrin (PE) (1:100 dilutions). Unstained cells were used to distinguish between fluorescent positive and fluorescent negative populations. 7-AAD was added for $10 \mathrm{~min}$ prior to FACS analysis, which allowed for the discrimination of dead vs. live cells. All antibodies were purchased from Becton Dickinson (Franklin Lakes, NJ, USA).

Confocal microscopy analysis. Immunofluorescent double staining analysis was performed on ECDHCC and HUVECs which were seeded on sterile slice cover slips in six well plates overnight. Following several washes with PBS, cells were fixed with $4 \%$ paraformaldehyde for $30 \mathrm{~min}$ at room temperature and permeabilized with $0.1 \%$ Triton X-100 in PBS for 5 min. The cells were overlaid with $5 \%$ BSA for $30 \mathrm{~min}$, rinsed with PBS and incubated with a mixture of rabbit-anti-human CD105 (1:20 dilutions; Abcam, Cambridge, UK) and mouse-anti-human CD31 (1:40 dilutions; DAKO) or a mixture of rabbit-anti-human CD105 (1:20 dilutions) and mouse-anti-human VEGFR-2 (1:200 dilutions) (both from Abcam) overnight at $4^{\circ} \mathrm{C}$. Cells were washed three times with $\mathrm{PBS}$ and then incubated with Alex-488-conjugated donkey-anti-rabbit IgG and Dylight-649-conjugated donkey-anti-mouse IgG (1:200 dilutions, Jackson ImmunoResearch, West Grove, PA, USA) secondary antibodies. The slices were incubated at $37^{\circ} \mathrm{C}$ for $45 \mathrm{~min}$, and nuclear staining was performed with DAPI. Coverslips were mounted with fluorescent mounting medium onto glass slides, and examined with confocal microscopy (Leica TCS SP5II; Leica Microsystems, Wetzlar, Germany). Images were collected from at least three independent experiments and processed for presentation in figures using Adobe Photoshop 6.0 (Adobe Systems, San Jose, CA, USA).

Statistical analysis. The stata17 software package was used for all statistical analyses. Comparisons between paired samples were determined by Wilcoxon signed-rank test. For the quantitative analysis of endothelial expression, one way ANOVA with post hoc test (Student-Newman-Keuls) was used for multiple comparisons of the merged areas covering CD105 with CD31 or VEGFR2. For clinical data analysis, a Chi-square test was used. A P-value of $<0.05$ was considered statistically significant. 


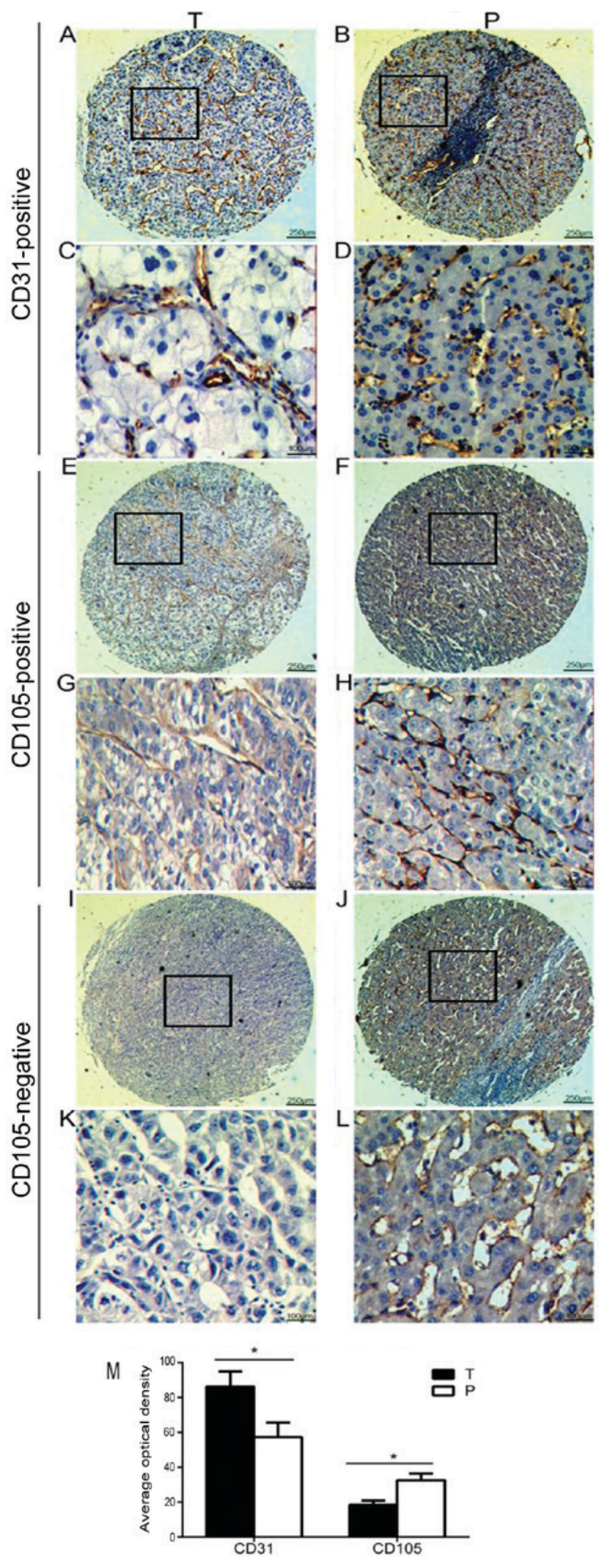

Figure 2. Tumor vessels with or without CD105 expression on TMA of HCC. (A-D) Representative images of CD31 expression in the T and $\mathrm{P}$ tissues ( $\mathrm{n}=90$ pairs). (E-H) Representative images of CD105-positive expression in T and $\mathrm{P}$ tissues ( $\mathrm{n}=51$ pairs). (I-L) Representative images of CD105-negative expression in tumor (I and $\mathrm{K}$ ) and positive expression of CD105 in peritumoral ( $\mathrm{J}$ and $\mathrm{L}$ ) tissues ( $\mathrm{n}=39$ pairs). Magnification $\mathrm{x} 40$ for A, B, E, F, I, J images, x100 for C, D, G, H, K, L images of TMA. (M) Total average optical density of CD31 and CD105 expression in tumor and peritumoral tissues $(n=90$ pairs). ${ }^{*} \mathrm{P}<0.05$. T, tumor; $\mathrm{P}$, peritumoral.
Table II. Overall analysis of HCC blood vessels with or without CD31 and CD105 expression by TMA with IHC staining.

\begin{tabular}{lcccc}
\hline & & \multicolumn{2}{c}{ Expression (\%) } & \\
\cline { 3 - 4 } Marker & No. of cases & Negative & Positive & P-value \\
\hline CD105 & 90 & $39(43.3)$ & $51(56.7)$ & $<0.01$ \\
CD31 & 90 & $0(0)$ & $90(100)$ &
\end{tabular}

HCC, hepatocellular carcinoma; IHC, immunohistochemistry; TMA, tissue microarray; $n$, the number of the case.

Table III. Clinical implication of the 39 cases with negative expression of CD105.

\begin{tabular}{lcc}
\hline Cases & Well (\%) & Poor (\%) \\
\hline Total no. of cases & $10 / 90(11.1)$ & $29 / 90(32.2)$ \\
No. of CD105 ${ }^{\text {neg }}$ cases & $10 / 39(25.6)$ & $29 / 39(74.4)$ \\
\hline
\end{tabular}

Well, well-differentiation of HCC, in which the characteristics of cancer cells were that the ratio of nucleus/cytoplasm was slightly increased, and nuclear atypia was minimal; Poor, poor-differentiation of $\mathrm{HCC}$, in which the characteristics of cancer cells were that the ratio of nucleus/cytoplasm was obviously increased, and the nuclei was hyperchromasia and nuclear borders were irregular. CD105 ${ }^{\text {neg }}$, negative expression of CD105 on TMA slices. CD105 ${ }^{\text {neg }}$, negative expression of CD105 on tissue microarray slices; $\mathrm{HCC}$, hepatocellular carcinoma.

\section{Results}

Sections of FFPE tissues derived from patients with HCC lacked endothelial CD105 expression. As presented in Fig. 1 by IHC detection, CD31 was universally present in vessels of all tumor and corresponding peritumoral tissues (Fig. 1A). Similarly, CD105 presented in all peritumoral tissues. However, CD105 in tumor tissues was differentially expressed: Of 15 patients with HCC, 9 tumor FFPE tissues presented CD105 abundantly in the vessels and in some tumor cells (Fig. 1B); whereas 6 tumor FFPE tissues appeared to exhibit weak or negative CD105 expression (Fig. 1C). Quantitative analysis revealed the differences of intensity between CD31 and CD105 expression, as well as between tumor and peritumoral tissues. Notably, the intensity of CD105 in those 6 tumor FFPE tissues with little or no $\mathrm{CD} 105$ expression $(\mathrm{AOD}=5 \pm 2)$ was significantly decreased when compared with corresponding peritumoral tissues ( $\mathrm{AOD}=55 \pm 4$ ), $\mathrm{P}<0.01$ (Fig. 1D).

Furthermore, we qualitatively scored CD31 and CD105 expression in tumor vessels by TMA and found that among 90 patients with HCC, CD31 was expressed in tumor vessels of all TMA specimens including tumor and peritumoral areas (Fig. 2A-D and Table II), whereas in 39 out of 90 cases (43.3\%) CD105 expression was absent in tumors in contrast to corresponding peritumoral tissues in which CD105 was pres ent (Fig. 2E-L and Table II). In addition, the overall intensity of the CD105 signal in tumors (AOD=19.2 \pm 4.6 ) was markedly 

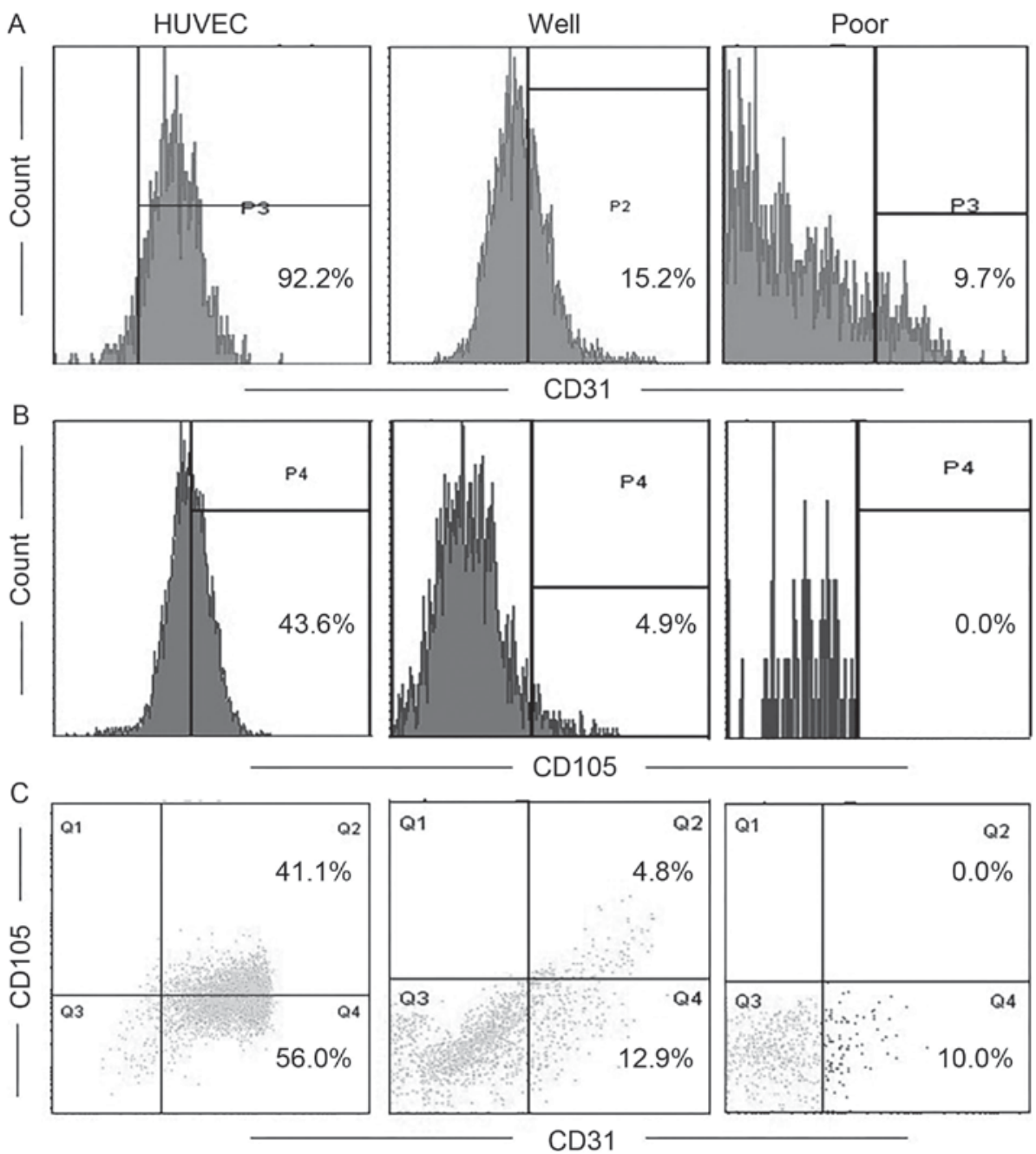

Figure 3. Flow cytometric analysis of expression levels of CD105 and CD31 in different HCC cases. Representative images of (A) CD31 and (B) CD105 staining in HUVECs (control), single cells from well- and poor-differentiated HCC, respectively. (C) Double staining of HUVECs, single cells from well- and poor-differentiated HCC with anti-CD31 and anti-CD105 antibodies.

low, compared with $\mathrm{CD} 31(\mathrm{AOD}=90 \pm 4.6)$ as well as with the intensity of $\mathrm{CD} 105$ in peritumor tissues $(\mathrm{AOD}=31.3 \pm 5.6)$ $(\mathrm{P}<0.05)$ (Fig. 2M). To investigate the clinical implication of CD105 expression variation, we retrospectively analyzed the clinicopathological data and found that of 39 CD105 negative cases, only 10 cases $(25.6 \%)$ were in the well-differentiated group, and the remaining 29 cases $(74.4 \%)$ were in the poor-differentiated group (Table III). Furthermore, the overall survival in the remaining 29 patients was significantly reduced (data not shown). In brief, the present results revealed that low or negative expression of CD105 in tumor vessels may indicate the deterioration of disease.

Variation in CD31 and CD105 expression levels at the cellular level, detected via flow cytometry and confocal microscopy analysis. In order to effectively conduct these assays to detect endothelial markers at the cellular level, a TEC single cell suspension was required. We respectively isolated TECs from 11 fresh resections from patients with HCC that were selected from the 15 aforementioned cases. Representative profiles from the flow cytometry analysis revealed that the CD31 expression level was 92.2, 15.2, and 9.7\% respectively in HUVECs, well- and poor-differentiated groups (Fig. 3A). CD105 expression was 43.6 and $4.9 \%$ in HUVECs and the well-differentiated group, respectively. A representative sample of patients with poor-differentiated HCC demonstrated undetectable levels of CD105 (Fig. 3B). $\mathrm{CD} 31^{+} \mathrm{CD} 105^{+}$double positive cells in $\mathrm{HCC}$ tissues decreased compared with in HUVECs (as a positive control) (Fig. 3C). The result indicated that the more advanced the stage of HCC, the lower the expression level of CD105.

Using 'double staining' in cells for confocal microscopy analysis makes it possible to detect co-localization of two endothelial makers in a cell. We performed 'double staining' in TEC (ECDHCC) and HUVECs (control) with 3 endothelial markers CD31, CD105 and VEGFR-2. As shown in Fig. 4, the representative images revealed that CD31 or VEGFR-2 were expressed abundantly in ECDHCC cells from all tested samples regardless of the degree of differentiation, in addition to HUVECs (Fig. 4A-F in the left 2 panels). However, CD105 positive expression presented in the well-differentiated cases ( $n=6 / 11$ ) only (Fig. 4B and E left panels), and little or no CD105 was expressed in the poor-differentiated cases $(n=5 / 11)$ (Fig. 4C and F left panels). On investigation of co-expression of CD105 with other endothelial markers, it was observed that an increased level of co-expression of CD105 with CD31 or 

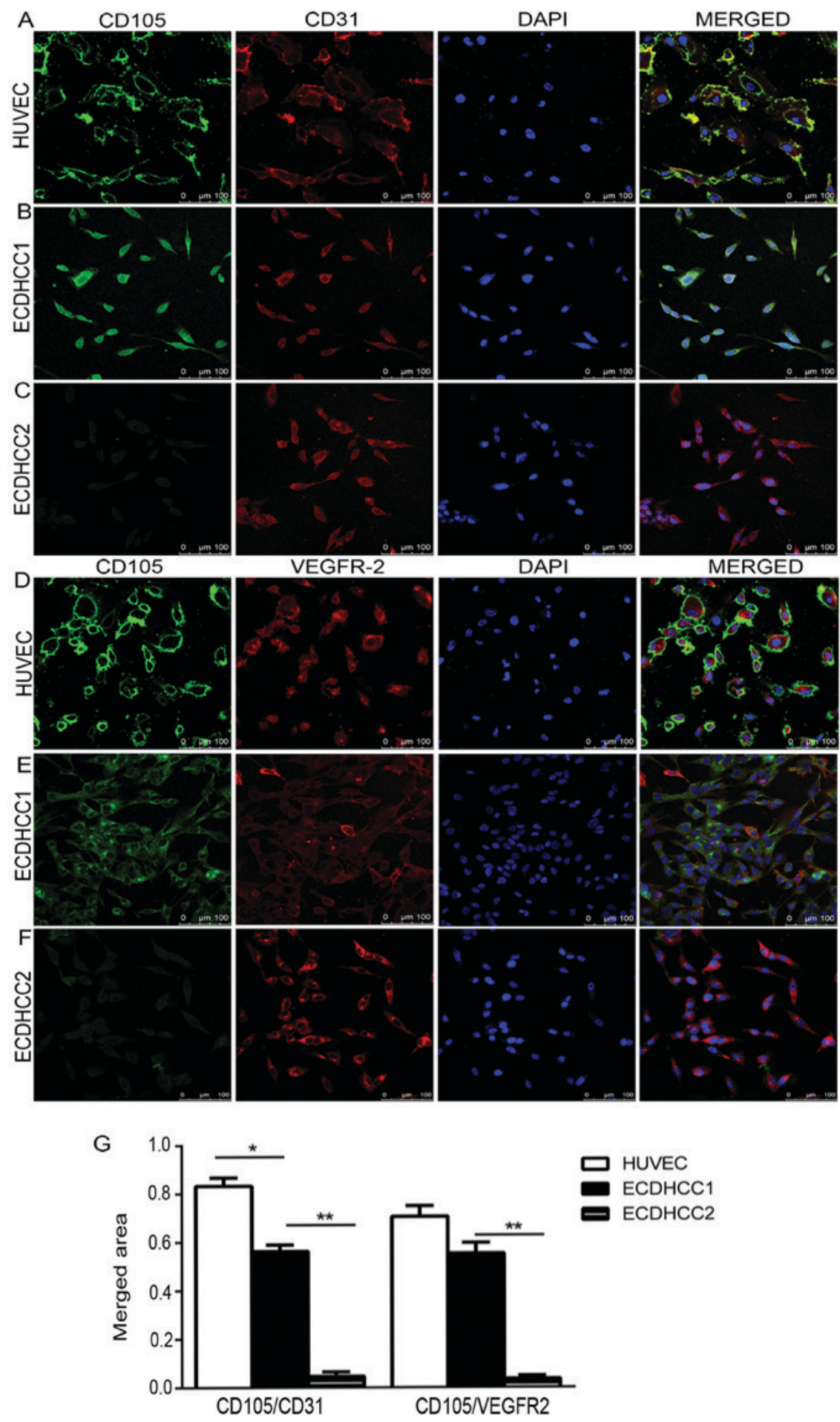

Figure 4. Confocal analysis of co-expression of CD105 with other endothelial markers in ECDHCC cells. Double staining of HUVECs (control) (A), ECDHCC1 (well-differentiated HCC, n=6) (B) and ECDHCC2 (poor-differentiated HCC, n=5) (C) with anti-CD105 and anti-CD31 antibodies. Double staining of HUVECs (D), ECDHCC1 (E) and ECDHCC2 (F) with anti-CD105 and anti-VEGFR-2 antibodies. (G) Quantitative analysis of the merged area covering CD105 with CD31 or VEGFR2. Anti-CD105, -CD31 and -VEGFR-2 antibodies were respectively labelled with Alex-488 (green) and Dylight-649 (red). Magnification $\mathrm{x} 400 .{ }^{*} \mathrm{P}<0.05,{ }^{* *} \mathrm{P}<0.01$.

VEGFR-2 appeared in HUVECs with $83.3 \pm 5.2$ and $70.7 \pm 3.9 \%$ of cover area (Fig. 4A-D, right panels and 4G). In the well-differentiated group, the merged areas of CD105 co-expressed with CD31 or VEGFR-2 were $56.8 \pm 2.6$ and $55.3 \pm 4.8 \%$ (Fig. 4B-E, right panels and $4 \mathrm{G}$ ). Whereas there was little or no merged area in the poor-differentiated group (Fig. 4C-F, right panels). The difference of the merged areas of CD105 with CD31 or
VEGFR-2 between the well- and poor-differentiated group was significant $(\mathrm{P}<0.01)$ (Fig. 4G).

\section{Discussion}

Antiangiogenic therapies aim to inhibit tumor angiogenesis by targeting the tumor blood vessels and lead to a 'starvation 
effect' on tumors and are extensively applied in clinical solid cancer treatment $(21,22)$. However, it has recently been noted that the efficiency of current antiangiogenic cancer therapy is limited and the treatment outcomes are different. This may potentially be due to the complex process of tumor angiogenesis, that leads to heterogeneity of tumor microvascular structures, including T-MAP (2), and multiple cell sources of recruitment for tumor angiogenesis, including cancer stem-like cells $(23,24)$, bone marrow-derived endothelial and hematopoietic precursor cells (25). Current drugs targeting tumor angiogenesis have been developed based on studies using normal ECs. Thus, it is necessary to isolate endothelial cells from tumor tissue for further understanding and improved treatment options and specificity.

Recently, several laboratories have isolated TECs using a variety of methods, however MACS is a far more specific method. Typically, endothelial markers including CD31, CD34 and CD105 are used for making antibodies that are bound with the magnetic beads, so that the cells expressing these markers can be positively selected. However, it is a controversial issue as to which type of endothelial molecule, as a stable marker, can be used for isolation of TECs. CD31 is known as a pan endothelial marker $(26,27)$. In our previous study, we phenotypically and functionally identified CD $31^{+}$TECs from human HCC mass (unpublished data). CD34 has also been used for isolation of TEC cells (28), however is primarily expressed in hematopoietic stem/progenitor cells (29). CD105 not only serves as an endothelial marker $(11,30)$ but is also expressed in tumor cells and thus may act as a prognosis marker for cancer $(13,31)$. These results indicate that the cells isolated using CD105-MACS contain both endothelial $\mathrm{CD}_{105^{+}}$and tumoral $\mathrm{CD} 105^{+}$cells.

The present study demonstrated that all 90 HCC-TMA cases exhibited CD31 expression in tumor tissue spots, whereas of these samples 39 HCC-TMA cases exhibited little or no CD105 expression in tumors, however this was not the case in peritumoral tissue spots. Of the 39 cases with negative CD105 expression, 29 cases $(74.4 \%)$ were poor-differentiated HCC. Interestingly, we found that the more advanced the stage of HCC, the lower the expression level of CD105. These findings were further verified by FACS analysis of CD31 and CD105 expression levels in a single cell digested from 11 HCC tissues, and by confocal analysis of TECs isolated from the same 11 HCC tissues. We found that HCC with poor differentiation did not express CD105. Similar to our data, it was demonstrated that CD105 has a lower expression in HCC compared with tumor free tissues, by MVD-CD105 (13). CD105 was also demonstrated to be completely negative in 28 cases of $86 \mathrm{HCC}$ sections examined (17). Conversely, endothelial CD105 has a high expression in a wide range of cancers, including colon, breast, brain, lung, prostate and cervical (4).

On comparison of CD105 with CD31 in tumor vessels of HCC, our study demonstrated that there might be a limitation to use CD105 as an endothelial marker for isolation of TECs, particularly in poor-differentiated HCC cases, due to lacking CD105 expression. In addition, there might be a potential risk of a contamination with $\mathrm{CD} 105^{+}$tumor cells. We believe that CD31, and not CD105 is a reliable endothelial marker for isolation of TECs. Further research is required in order to clarify the potential mechanisms.

\section{Acknowledgements}

The authors would like to thank Dr Runhua Luo (Cancer Research Center Nantong, Tumor Hospital Affiliated to Nantong University, China) for technical assistance.

\section{Funding}

The present study was supported by the National Natural Science Foundation of China (grant no. 81272378), Nantong Science and Technology Bureau (grant no. MS22015005), Tumor Hospital Affiliated to Nantong University (grant no. YY201211) and by the Nantong Medical Young Talents grant (grant no. 2017.33).

\section{Availability of data and materials}

All data generated or analyzed during this study are included in this published article.

\section{Authors' contributions}

HQ and WZ were involved in acquisition and analysis of confocal and flow cytometry data. $\mathrm{HC}$ and $\mathrm{SH}$ were involved in the collection of human tissues. HQ was involved in interpretation of the data. LY was involved in the conception and design of the present study. All authors read and approved the final manuscript.

\section{Ethics approval and consent to participate}

The study was approved by Ethics Committee of Tumor Hospital Affiliated to Nantong University (no. 2015-0067). Written informed consent was signed and obtained from all the patients who were involved in this study.

\section{Patient consent for publication}

Not applicable.

\section{Competing interests}

All authors declare that there are no competing interests.

\section{References}

1. Holleb AI and Folkman J: Tumor angiogenesis. CA Cancer J Clin 22: 226-229, 1972.

2. Bian XW, Wang QL, Xiao HL and Wang JM: Tumor microvascular architecture phenotype (T-MAP) as a new concept for studies of angiogenesis and oncology. J Neurooncol 80: 211-213, 2006.

3. Ohga N, Ishikawa S, Maishi N, Akiyama K, Hida Y, Kawamoto T, Sadamoto Y, Osawa T, Yamamoto K, Kondoh M, et al: Heterogeneity of tumor endothelial cells: Comparison between tumor endothelial cells isolated from high- and low-metastatic tumors. Am J Pathol 180: 1294-1307, 2012.

4. Duff SE, Li C, Garland JM and Kumar S: CD105 is important for angiogenesis: Evidence and potential applications. FASEB J 17: 984-992, 2003.

5. Mahajan KD, Nabar GM, Xue W, Anghelina M, Moldovan NI, Chalmers JJ and Winter JO: Mechanotransduction effects on endothelial cell proliferation via CD31 and VEGFR2: Implications for immunomagnetic separation. Biotechnol J 12, 2017. 
6. Qin M, Guan X, Wang H, Zhang Y, Shen B, Zhang Q, Dai W, Ma Y and Jiang Y: An effective ex vivo approach for inducing endothelial progenitor cells from umbilical cord blood CD34 cells. Stem Cell Res Ther 8: 25, 2017.

7. Xiong YQ, Sun HC, Zhang W, Zhu XD, Zhuang PY, Zhang JB, Wang L, Wu WZ, Qin LX and Tang ZY: Human hepatocellular carcinoma tumor-derived endothelial cells manifest increased angiogenesis capability and drug resistance compared with normal endothelial cells. Clin Cancer Res 15: 4838-4846, 2009.

8. Afshar Moghaddam N, Mahsuni P and Taheri D: Evaluation of endoglin as an angiogenesis marker in glioblastoma. Iran J Pathol 10: 89-96, 2015.

9. Davidson B, Stavnes HT, Førsund M, Berner A and Staff AC: CD105 (Endoglin) expression in breast carcinoma effusions is a marker of poor survival. Breast 19: 493-498, 2010.

10. Fonsatti E, Nicolay HJ, Altomonte M, Covre A and Maio M: Targeting cancer vasculature via endoglin/CD105: A novel antibody-based diagnostic and therapeutic strategy in solid tumours. Cardiovasc Res 86: 12-19, 2010.

11. Sakurai T, Okumura H, Matsumoto M, Uchikado Y, Owaki T, Kita Y, Setoyama T, Omoto I, Kijima Y, Ishigami S, et al: Endoglin (CD105) is a useful marker for evaluating microvessel density and predicting prognosis in esophageal squamous cell carcinoma. Anticancer Res 34: 3431-3438, 2014.

12. Martinez LM, Labovsky V, Calcagno Mde L, Davies KM, Rivello HG, Wernicke A, Calvo JC and Chasseing NA: Comparative prognostic relevance of breast intra-tumoral microvessel density evaluated by CD105 and CD146: A pilot study of 42 cases. Pathol Res Pract 212: 350-355, 2016.

13. Saroufim A, Messai Y, Hasmim M, Rioux N, Iacovelli R, Verhoest G, Bensalah K, Patard JJ, Albiges L, Azzarone B, et al: Tumoral CD105 is a novel independent prognostic marker for prognosis in clear-cell renal cell carcinoma. Br J Cancer 110: $1778-1784,2014$.

14. Yao Y, Pan Y, Chen J, Sun X, Qiu Y and Ding Y: Endoglin (CD105) expression in angiogenesis of primary hepatocellular carcinomas: Analysis using tissue microarrays and comparisons with CD34 and VEGF. Ann Clin Lab Sci 37: 39-48, 2007.

15. Chen CH, Chuang HC, Lin YT, Fang FM, Huang CC, Chen CM, $\mathrm{Lu} \mathrm{H}$ and Chien CY: Circulating CD105 shows significant impact in patients of oral cancer and promotes malignancy of cancer cells via CCL20. Tumour Biol 37: 1995-2005, 2016.

16. Nair S, Nayak R, Bhat $K$, Kotrashetti VS and Babji D: Immunohistochemical expression of CD105 and TGF- $\beta 1$ in oral squamous cell carcinoma and adjacent apparently normal oral mucosa and its correlation with clinicopathologic features. Appl Immunohistochem Mol Morphol 24: 35-41, 2016.

17. HoJW,Poon RT,Sun CK, Xue WC and Fan ST: Clinicopathological and prognostic implications of endoglin (CD105) expression in hepatocellular carcinoma and its adjacent non-tumorous liver. World J Gastroenterol 11: 176-181, 2005.

18. Li C, Hampson IN, Hampson L, Kumar P, Bernabeu C and Kumar S: CD105 antagonizes the inhibitory signaling of transforming growth factor beta1 on human vascular endothelial cells. FASEB J 14: 55-64, 2000.

19. Li C, Issa R, Kumar P, Hampson IN, Lopez-Novoa JM, Bernabeu C and Kumar S: CD105 prevents apoptosis in hypoxic endothelial cells. J Cell Sci 116: 2677-2685, 2003.
20. Hewett PW: Isolation and culture of human endothelial cells from micro- and macro-vessels. Methods Mol Biol 1430: 61-76, 2016.

21. Siegel AB, Cohen EI, Ocean A, Lehrer D, Goldenberg A, Knox JJ, Chen H, Clark-Garvey S, Weinberg A, Mandeli J, et al: Phase II trial evaluating the clinical and biologic effects of bevacizumab in unresectable hepatocellular carcinoma. J Clin Oncol 26: 2992-2998, 2008.

22. Gutierrez M and Giaccone G: Antiangiogenic therapy in nonsmall cell lung cancer. Curr Opin Oncol 20: 176-182, 2008.

23. Ricci-Vitiani L, Pallini R, Biffoni M, Todaro M, Invernici G, Cenci T, Maira G, Parati EA, Stassi G, Larocca LM, et al: Tumour vascularization via endothelial differentiation of glioblastoma stem-like cells. Nature 468: 824-828, 2010.

24. Wang R, Chadalavada K, Wilshire J, Kowalik U, Hovinga KE, Geber A, Fligelman B, Leversha M, Brennan C and Tabar V: Glioblastoma stem-like cells give rise to tumour endothelium. Nature 468: 829-833, 2010.

25. Lyden D, Hattori K, Dias S, Costa C, Blaikie P, Butros L, Chadburn A, Heissig B, Marks W, Witte L, et al: Impaired recruitment of bone-marrow-derived endothelial and hematopoietic precursor cells blocks tumor angiogenesis and growth. Nat Med 7: 1194-1201, 2001.

26. Castilho-Fernandes A, de Almeida DC, Fontes AM, Melo FU, Picanço-Castro V, Freitas MC, Orellana MD, Palma PV, Hackett PB, Friedman SL, et al: Human hepatic stellate cell line (LX-2) exhibits characteristics of bone marrow-derived mesenchymal stem cells. Exp Mol Pathol 91: 664-672, 2011.

27. Rubatt JM, Darcy KM, Hutson A, Bean SM, Havrilesky LJ, Grace LA, Berchuck A and Secord AA: Independent prognostic relevance of microvessel density in advanced epithelial ovarian cancer and associations between CD31, CD105, p53 status, and angiogenic marker expression: A Gynecologic Oncology Group study. Gynecol Oncol 112: 469-474, 2009.

28. Miyata Y, Mitsunari K, Asai A, Takehara K, Mochizuki Y and Sakai H: Pathological significance and prognostic role of microvessel density, evaluated using CD31, CD34, and CD105 in prostate cancer patients after radical prostatectomy with neoadjuvant therapy. Prostate 75: 84-91, 2015.

29. Yang L, Bryder D, Adolfsson J, Nygren J, Månsson R, Sigvardsson M and Jacobsen SE: Identification of Lin(-)Sca1(+) kit(+)CD34(+)Flt3-short-term hematopoietic stem cells capable of rapidly reconstituting and rescuing myeloablated transplant recipients. Blood 105: 2717-2723, 2005.

30. Zhou Y, Gu H, Xu Y, Li F, Kuang S, Wang Z, Zhou X, Ma H, Li P, Zheng $\mathrm{Y}$, et al: Targeted antiangiogenesis gene therapy using targeted cationic microbubbles conjugated with CD105 antibody compared with untargeted cationic and neutral microbubbles. Theranostics 5: 399-417, 2015.

31. Li Y, Zhai Z, Liu D, Zhong X, Meng X, Yang Q, Liu J and Li H: CD105 promotes hepatocarcinoma cell invasion and metastasis through VEGF. Tumour Biol 36: 737-745, 2015.

This work is licensed under a Creative Commons Attribution-NonCommercial-NoDerivatives 4.0 International (CC BY-NC-ND 4.0) License. 